\title{
Two-Year Outcomes after Left Main Coronary Artery Percutaneous Coronary Intervention in Patients Presenting with Acute Coronary Syndrome
}

\author{
Si-Da Jia, Yi Yao, Ying Song, Xiao-Fang Tang, Xue-Yan Zhao, Run-Lin Gao, Yue-Jin Yang, \\ Bo Xu $(\mathbb{D}$, Zhan Gao, and Jin-Qing Yuan $\mathbb{D}$
}

Fu Wai Hospital, National Center for Cardiovascular Diseases,

Peking Union Medical College and Chinese Academy of Medical Sciences, Beijing, China

Correspondence should be addressed to Jin-Qing Yuan; dr_jinqingyuan@sina.com

Received 22 September 2019; Accepted 10 March 2020; Published 7 April 2020

Academic Editor: Amit Segev

Copyright ( $2020 \mathrm{Si}$-Da Jia et al. This is an open access article distributed under the Creative Commons Attribution License, which permits unrestricted use, distribution, and reproduction in any medium, provided the original work is properly cited.

\begin{abstract}
Objectives. We aim to evaluate long-term outcomes after left main coronary artery (LMCA) percutaneous coronary intervention (PCI) in patients presenting with acute coronary syndrome (ACS). Background. PCI of the LMCA has been an acceptable revascularization strategy in stable coronary artery disease. However, limited studies on long-term clinical outcomes of LMCA PCI in ACS patients are available. Methods. A total of 6429 consecutive patients with ACS undergoing PCI in Fuwai Hospital in 2013 were enrolled. Patients are divided into LMCA group and Non-LMCA group according to whether the target lesion was located in LMCA. Prognosis impact on 2-year major adverse cardiovascular and cerebrovascular events (MACCE) is analyzed. Results. 155 (2.4\%) patients had target lesion in LMCA, while 6274 (97.6\%) patients belong to the non-LMCA group. Compared with non-LMCA patients, LMCA patients have generally more comorbidities and worse baseline conditions. Two-year follow-up reveals that LMCA patients have significantly higher rate of cardiac death $(2.6 \%$ vs. $0.7 \%, p=0.034)$, myocardial infarction $(7.1 \%$ vs. $1.8 \%, p<0.001)$, in-stent thrombosis $(4.5 \%$ vs. $0.8 \%, p<0.001)$, and stroke $(7.1 \%$ vs. $6.4 \%, p=0.025)$. After adjusting for confounding factors, LMCA remains independently associated with higher 2-year myocardial infarction rate $(\mathrm{HR}=2.585,95 \%$ $\mathrm{CI}=1.243-5.347, p=0.011$ ). Conclusion. LMCA-targeted PCI is an independent risk factor for 2-year myocardial infarction in ACS patients.
\end{abstract}

\section{Introduction}

For patients with low SYNergy between percutaneous coronary intervention with TAXus and cardiac surgery (SYNTAX) score, percutaneous coronary intervention (PCI) has been recommended as a reasonable revascularization strategy in patients with significant stenosis in the left main coronary artery (LMCA) presenting with stable coronary artery disease (SCAD) [1]. Previous studies have proven that, compared with coronary artery bypass graft (CABG) surgeries, unprotected LMCA-targeted PCI resulted in similar rate of mortality and composite event of death, myocardial infarction (MI), and stroke [2-10]. However, studies on long-term clinical outcomes of LMCA PCI in acute coronary syndrome (ACS) patients are relatively rare.
A limited number of studies have yielded conflicting results in terms of LMCA PCI in ACS settings. Several studies found that, although patients with AMI and thrombosis in unprotected LMCA are at high-risk for substantial mortality, PCI is still associated with a remarkably high short-term and long-term survival rates [11-13]. Moreover, in another study reported by Gao et al. [14], transradial PCI on unprotected LMCA and/or multivessel disease for patients with ACS had comparable clinical outcomes to CABG, with an advantage of reducing stroke. Contrarily, Baek et al. [15] found patients with ST elevation myocardial infarction (STEMI) and LMCA PCI had poor clinical outcome, which is attributable to periprocedural hemodynamic deterioration. A recent analysis from the EXCEL trial has found that patients with LMCA disease 
undergoing PCI or CABG had similar rate of adverse events irrespective of the acuity of clinical presentation [16]. However, these studies were modest in sample size or failed to observe the long-term outcome of LMCA PCI in realworld clinical settings.

Thus, we aim to evaluate long-term clinical outcome of LMCA PCI in patients presenting with ACS in our realworld, prospective, large-sample cohort of Chinese patients.

\section{Materials and Methods}

2.1. Study Population. Data from all consecutive patients from a single center (Fu Wai Hospital, National Center for Cardiovascular Diseases, Beijing, China) undergoing PCI were prospectively collected. Based on contemporary practice guidelines, revascularization strategies were finally determined by heart team discussion involving interventional cardiologists, cardiac surgeons, and physicians. Patients who did not undergo PCI and were referred for CABG after heart team discussion were excluded from this study. Between January 2013 and December 2013, a total of 10,724 consecutive patients were enrolled undergoing PCI. The Institutional Review Board approved the study protocol, and the patients provided written informed consent before the intervention.

Exclusion criteria included patients presenting with $\operatorname{SCAD}(n=4,295)$. Thus, 6,429 ACS patients undergoing PCI were included in the present study. Patients presenting with ACS and LMCA stenosis requiring percutaneous coronary intervention were included in the LMCA group, while other patients enrolled in this study were stratified into the nonLMCA group.

2.2. Procedure and Medications. The PCI strategy and stent type were left to treating physician's discretion. ACS patients (STEMI and NSTE-ACS) scheduled for PCI received the same dose aspirin and ticagrelor or clopidogrel (loadingdose $300 \mathrm{mg}$ or $600 \mathrm{mg}$ ) as soon as possible. During the procedure, unfractionated heparin $(100 \mathrm{U} / \mathrm{kg})$ was administered to all patients, and use of glycoprotein IIb/IIIa inhibitors was per operator's judgment. After the procedure, aspirin was prescribed at a dose of $100 \mathrm{mg}$ daily indefinitely; clopidogrel $75 \mathrm{mg}$ daily or ticagrelor $90 \mathrm{mg}$ twice daily was advised for at least 1 year after PCI.

2.3. Patient Follow-Up. All patients were evaluated by clinic visit or by phone at 1,6 , and 12 months and annually thereafter. Patients were advised to return for coronary angiography if clinically indicated by symptoms or documentation of myocardial ischemia.

2.4. Endpoints and Definitions. Death resulting from any reason, including cardiac death, was defined as all-cause death. Death that could not be attributed to a noncardiac etiology was considered cardiac death. MI was defined by the third universal definition of MI [17]. Revascularization was defined as repeated revascularization for ischemic symptoms and events driven by PCI or surgery of any vessel. Unplanned target vessel revascularization (TVR) was defined as repeat percutaneous intervention or surgical bypass of any segment of the target vessel for ischemic symptoms and events driven [18]. Stent thrombosis (ST) was defined according to the Academic Research Consortium, including definite, probable, and possible in the analysis [18]. Bleeding was quantified according to Bleeding Academic Research Consortium (BARC) definition criteria, including types 2, 3, and 5 in the analysis [19]. Major adverse cardiac and cerebrovascular event (MACCE) was defined as the occurrence of death, MI, TVR, ST, and stroke during follow-up. All endpoints were adjudicated centrally by 2 independent cardiologists, and disagreement was resolved by consensus. The SYNTAX score was assessed by two of three experienced cardiologists in an independent angiographic core lab and were blinded to the clinical outcomes. In case of disagreement among the two observers, opinion from the third cardiologist was obtained to finally reach a consensus.

2.5. Statistical Analysis. Continuous variables are expressed as mean \pm standard deviation, and categorical variables are presented as percentages. Differences in baseline characteristics and in-hospital outcomes between groups were assessed using the chi-squared test or Fisher's exact test for categorical variables and Student's $t$-test or the Wilcoxon rank test for continuous variables, as appropriate. Survival curves were constructed using Kaplan-Meier method, and the log-rank test was performed to compare the time to clinical endpoints. Cox regression analyses were conducted to evaluate the adjusted effect of LMCA PCI on 2-year clinical endpoints. Clinically and statistically significant covariates were all entered into the model, and results were reported as adjusted hazard ratios together with corresponding $95 \%$ confidence intervals (CI). Adjusted confounding factors include LMCA, age, diabetes, hemoglobin, GFR, STEMI, UA, preprocedural SYNTAX score, puncture site, staged PCI, IVUS, IABP, successful PCI, and stent type. For all analyses, a 2 -sided $p$ value $<0.05$ was considered significant. Statistical analysis was performed using IBM ${ }^{\circledR}$ SPSS ${ }^{\circledR}$ v22.0.0.0 software (SPSS Inc., Chicago, IL, USA).

To minimize the effect of confounding factors caused by differences in baseline characteristics between LMCA and non-LMCA groups, and propensity score match (PSM) was performed. A propensity score was estimated for each patient using a logistic regression model. Patients were matched on estimated propensity scores, using a nearest neighbor approach. The matched variables were LMCA, age, diabetes, hemoglobin, GFR, STEMI, UA, preprocedural SYNTAX score, puncture site, staged PCI, IVUS, IABP, successful PCI, and stent type.

\section{Results}

Among 6,429 patients presenting with ACS undergoing PCI, $155(2.4 \%)$ patients was in the LMCA group, while 6,274 (97.4\%) patients belonged to the non-LMCA group. Median follow-up time was 760 days. 147 (94.8\%) of patients in the 
TABLE 1: Baseline patient characteristics.

\begin{tabular}{|c|c|c|c|c|c|c|}
\hline & & efore PSM & & & After PSM & \\
\hline & $\begin{array}{l}\text { LMCA } \\
(n=155)\end{array}$ & $\begin{array}{c}\text { Non-LMCA } \\
(n=6274)\end{array}$ & $p$ value & $\begin{array}{l}\text { LMCA } \\
(n=150)\end{array}$ & $\begin{array}{c}\text { Non-LMCA } \\
(n=150)\end{array}$ & $p$ value \\
\hline Age & $61.91 \pm 9.80$ & $58.29 \pm 10.41$ & $<0.001$ & $61.96 \pm 9.68$ & $60.97 \pm 11.02$ & 0.411 \\
\hline Female, $n(\%)$ & $40(25.8)$ & $1452(23.1)$ & 0.438 & $39(26.0)$ & $41(27.3)$ & 0.794 \\
\hline Body mass index, $\mathrm{kg} / \mathrm{m}^{2}$ & $25.58 \pm 2.98$ & $25.89 \pm 3.20$ & 0.233 & $25.64 \pm 2.98$ & $25.41 \pm 3.58$ & 0.545 \\
\hline $\begin{array}{l}\text { Risk factors and history, } n(\%) \\
\text { Smoker } \\
\text { Diabetes } \\
\text { Hypertension } \\
\text { Hyperlipidemia } \\
\text { Prior myocardial infarction } \\
\text { Prior stroke }\end{array}$ & $\begin{array}{c}89(57.4) \\
56(36.1) \\
92(59.4) \\
101(65.2) \\
27(17.4) \\
20(12.9) \\
\end{array}$ & $\begin{array}{l}3755(59.9) \\
1791(28.5) \\
3993(63.6) \\
4125(65.7) \\
828(13.2) \\
674(10.7)\end{array}$ & $\begin{array}{l}0.542 \\
\mathbf{0 . 0 3 9} \\
0.273 \\
0.879 \\
0.126 \\
0.392 \\
\end{array}$ & $\begin{array}{l}86(57.3) \\
55(36.7) \\
89(59.3) \\
98(65.3) \\
26(17.3) \\
19(12.7) \\
\end{array}$ & $\begin{array}{c}79(52.7) \\
52(34.7) \\
95(63.3) \\
105(70.0) \\
18(12.0) \\
19(12.7) \\
\end{array}$ & $\begin{array}{l}0.417 \\
0.718 \\
0.477 \\
0.388 \\
0.192 \\
1.000 \\
\end{array}$ \\
\hline $\begin{array}{l}\text { Laboratory tests } \\
\text { Leukocyte, } \times 10^{9} / \mathrm{L} \\
\text { Platelet, } \times 10^{9} / \mathrm{L} \\
\text { Hemoglobin, g/L } \\
\text { Creatinine, } \mu \mathrm{mol} / \mathrm{L} \\
\text { GFR, } \mathrm{ml} / \mathrm{min} \\
\text { LVEF, } \%\end{array}$ & $\begin{array}{c}7.22 \pm 1.97 \\
209.97 \pm 59.71 \\
134.16 \pm 14.73 \\
76.55 \pm 18.90 \\
87.71 \pm 16.22 \\
62.82 \pm 6.95\end{array}$ & $\begin{array}{c}7.08 \pm 2.10 \\
208.51 \pm 57.02 \\
140.83 \pm 16.15 \\
76.03 \pm 16.41 \\
91.03 \pm 15.54 \\
62.28 \pm 7.46\end{array}$ & $\begin{array}{c}0.430 \\
0.757 \\
<\mathbf{0 . 0 0 1} \\
0.697 \\
\mathbf{0 . 0 0 9} \\
0.375\end{array}$ & $\begin{array}{c}7.22 \pm 1.97 \\
209.97 \pm 59.71 \\
134.16 \pm 14.73 \\
76.40 \pm 18.94 \\
87.79 \pm 16.36 \\
62.96 \pm 6.85\end{array}$ & $\begin{array}{c}7.27 \pm 2.21 \\
211.13 \pm 58.61 \\
134.73 \pm 15.71 \\
76.95 \pm 17.96 \\
88.29 \pm 17.44 \\
60.83 \pm 9.48\end{array}$ & $\begin{array}{l}0.828 \\
0.865 \\
0.745 \\
\mathbf{0 . 0 4 6} \\
0.799 \\
\mathbf{0 . 0 2 8}\end{array}$ \\
\hline $\begin{array}{l}\text { Clinical presentation } \\
\text { STEMI } \\
\text { NSTEMI } \\
\text { UA }\end{array}$ & $\begin{aligned} & 24(15.5) \\
& 11(7.1) \\
& 120(77.4) \\
&\end{aligned}$ & $\begin{aligned} & 1421(22.6) \\
& 464(7.4) \\
& 4389(70.0) \\
&\end{aligned}$ & $\begin{array}{l}\mathbf{0 . 0 3 5} \\
0.888 \\
\mathbf{0 . 0 4 5}\end{array}$ & $\begin{array}{c}23(15.3) \\
10(6.7) \\
117(78.0) \\
\end{array}$ & $\begin{array}{c}36(24.0) \\
12(8.0) \\
102(68.0) \\
\end{array}$ & $\begin{array}{l}0.059 \\
0.658 \\
0.051\end{array}$ \\
\hline $\begin{array}{l}\text { Medication at discharge, } n(\%) \\
\text { Aspirin } \\
\text { Clopidogrel } \\
\text { Ticagrelor } \\
\beta \text {-blockers } \\
\text { Calcium channel blockers } \\
\text { Nitrates } \\
\text { Statins }\end{array}$ & $\begin{array}{c}154(99.4) \\
154(99.4) \\
1(0.6) \\
146(94.2) \\
80(51.6) \\
154(99.4) \\
150(96.8)\end{array}$ & $\begin{array}{c}6185(98.6) \\
6258(99.7) \\
13(0.2) \\
5575(88.9) \\
3121(49.7) \\
6141(97.9) \\
6011(95.8)\end{array}$ & $\begin{array}{l}0.726 \\
0.340 \\
0.290 \\
\mathbf{0 . 0 3 6} \\
0.646 \\
0.382 \\
0.552\end{array}$ & $\begin{array}{c}149(99.3) \\
149(99.3) \\
1(0.7) \\
142(94.7) \\
76(50.7) \\
149(99.3) \\
146(95.3)\end{array}$ & $\begin{array}{c}149(99.3) \\
149(99.3) \\
1(0.7) \\
141(94.0) \\
68(45.3) \\
147(98.0) \\
140(93.3)\end{array}$ & $\begin{array}{l}1.000 \\
1.000 \\
1.000 \\
0.803 \\
0.355 \\
0.622 \\
0.169\end{array}$ \\
\hline
\end{tabular}

Values are mean $\pm \mathrm{SD}$ or $n(\%)$. GFR = glomerular filtration rate; $\mathrm{LVEF}=$ left ventricular ejection fraction; STEMI = ST elevation myocardial infarction; $\mathrm{NSTEMI}=$ non-ST elevation myocardial infarction; $\mathrm{UA}=$ unstable angina .

LMCA group had unprotected LMCA. Before PSM, compared with the non-LMCA group, patients in the LMCA group are older, with higher proportion of diabetes, more clinical presentation of unstable angina (UA), and $\beta$-blocker usage. Laboratory findings indicated lower levels of hemoglobin and glomerular filtration rate (GFR) for LMCA patients (Table 1). In terms of angiographic and procedural findings, LMCA patients are associated with higher preprocedural SYNTAX score, higher rate of trivessel disease, staged PCI, usage of intravascular ultrasound (IVUS) and intraaortic balloon pump (IABP) support, and fewer implantations of first-generation drug-eluting stents (1G-DES) (Table 2). After PSM, all matched baseline characteristics were no longer significantly different, despite introducing a few new imbalance in baseline and angiographic characteristics between groups.

Before PSM, follow-up results revealed that LMCA patients had higher incidence of 2 -year cardiac death $(2.6 \%$ vs. $0.7 \%, p=0.034)$, target vessel MI ( $5.2 \%$ vs. $0.8 \%, p=0.001)$, in-stent thrombosis $(4.5 \%$ vs. $0.8 \%, p=0.001)$, and stroke (3.9\% vs. $1.4 \%, p=0.025)$, while no difference was observed for all-cause death, target lesion MI, unplanned revascularization, bleeding, and MACCE (all $p>0.05$ ) (Table 3). Kaplan-Meier survival analysis revealed similar findings, except that no difference was observed for 2-year in-stent thrombosis-free survival (Figure 1). After PSM, the incidence of most clinical outcomes was nonsignificant between LMCA and non-LMCA groups, except for a significantly higher rate of MI in the LMCA group (3.0\% vs. $1.3 \%, p=0.011$ ).

Before PSM and after adjusting for differences by Cox regression analysis in age, diabetes, hemoglobin and GFR levels, clinical presentation, preprocedural SYNTAX score, puncture site, staged PCI, IVUS and IABP usage, successful PCI, and stent type, we found LMCA was independently associated with higher risk of 2-year MI ( $\mathrm{HR}=2.585,95 \%$ $\mathrm{CI}=1.243-5.347, \quad p=0.011)$ and in-stent thrombosis $(\mathrm{HR}=2.888,95 \% \mathrm{CI}=1.101-7.576, p=0.031)$. For all-cause death, cardiogenic death, unplanned revascularization, stroke, bleeding, and MACCE, the difference was nonsignificant (all $p>0.05)$. After PSM, however, only difference in occurrence of 2-year MI (HR $=10.992,95 \%$ CI: 2.000-60.417, $p=0.006$ ) remained statistically significant, while difference in 2-year instent thrombosis rate became nonsignificant (Figure 2).

Subgroup analysis revealed that LMCA patients presenting with STEMI had higher risk of 2-year all-cause death $(12.5 \%$ vs. $3.0 \%, p=0.013)$, cardiac death $(12.5 \%$ vs. $1.3 \%$, $p=0.005)$, MI $(16.7 \%$ vs. $2.2 \%, p=0.002)$, and in-stent 
TABLE 2: Coronary angiographic findings and percutaneous interventional therapies.

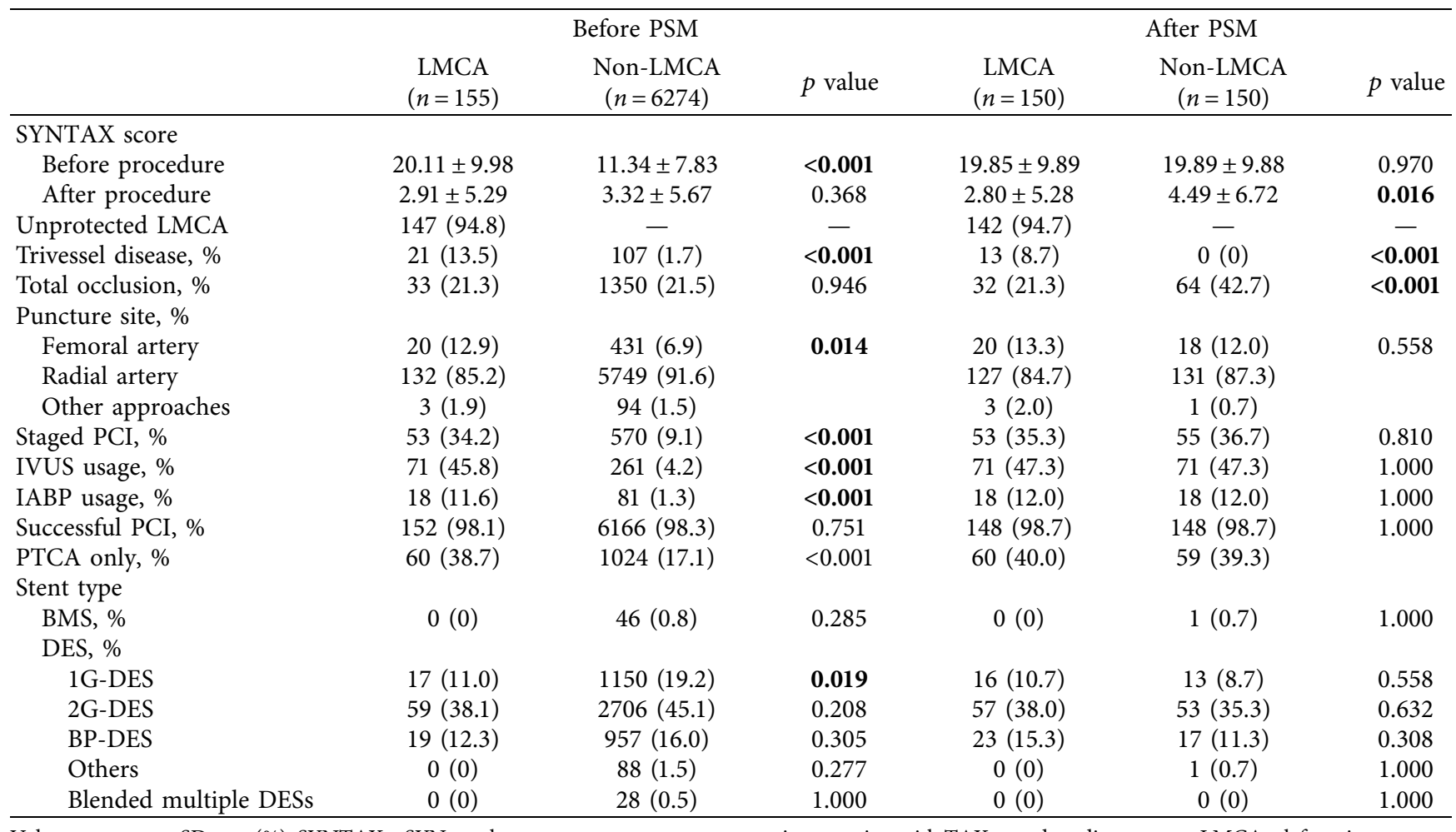

Values are mean \pm SD or $n(\%)$. SYNTAX = SYNergy between percutaneous coronary intervention with TAXus and cardiac surgery; LMCA = left main coronary artery; $\mathrm{PCI}=$ percutaneous coronary intervention; IVUS = intravascular ultrasound; $\mathrm{IABP}=$ intraaortic balloon pump; PTCA= percutaneous transluminal coronary angioplasty; $\mathrm{BMS}=$ bare metal stent; $\mathrm{DES}=$ drug-eluting stent; $1 \mathrm{G}=$ first generation; $2 \mathrm{G}=$ second generation; $\mathrm{BP}=$ biodegradable polymer.

TABLE 3: Two-year clinical outcomes.

\begin{tabular}{|c|c|c|c|c|c|c|}
\hline & \multicolumn{3}{|c|}{ Before PSM } & \multicolumn{3}{|c|}{ After PSM } \\
\hline & LMCA $(n=155)$ & Non-LMCA $(n=6274)$ & $p$ value & LMCA $(n=150)$ & Non-LMCA $(n=150)$ & $p$ value \\
\hline All-cause death & $4(2.6)$ & $82(1.3)$ & 0.153 & $3(2.0)$ & $2(1.3)$ & 1.000 \\
\hline Cardiac death & $4(2.6)$ & $47(0.7)$ & 0.034 & $3(2.0)$ & $0(0)$ & 0.247 \\
\hline Myocardial infarction & $11(7.1)$ & $116(1.8)$ & $<0.001$ & $11(3.0)$ & $2(1.3)$ & 0.011 \\
\hline Target vessel related & $8(5.2)$ & $52(0.8)$ & $<0.001$ & $8(5.3)$ & $3(2.0)$ & 0.125 \\
\hline Target lesion related & $2(1.3)$ & $31(0.5)$ & 0.188 & $2(1.3)$ & $1(0.7)$ & 1.000 \\
\hline Unplanned revascularization & $10(6.5)$ & $546(8.7)$ & 0.325 & $10(6.7)$ & $15(10.0)$ & 0.296 \\
\hline Target vessel related & $9(5.8)$ & $146(94.2)$ & 0.722 & $9(6.0)$ & $9(6.0)$ & 1.000 \\
\hline Target lesion related & $7(4.5)$ & $148(95.5)$ & 0.755 & $7(4.7)$ & $7(4.7)$ & 1.000 \\
\hline In-stent thrombosis & $7(4.5)$ & $51(0.8)$ & $<0.001$ & $6(4.0)$ & $2(1.3)$ & 0.282 \\
\hline Stroke & $6(3.9)$ & $88(1.4)$ & 0.025 & $5(3.3)$ & $6(4.0)$ & 0.759 \\
\hline Bleeding & $11(7.1)$ & $401(6.4)$ & 0.723 & $11(7.3)$ & $6(4.0)$ & 0.212 \\
\hline MACCE & $24(15.5)$ & $768(12.2)$ & 0.225 & $22(14.7)$ & $23(15.3)$ & 0.872 \\
\hline
\end{tabular}

$\mathrm{MACCE}=$ major adverse cardiac and cerebrovascular events.

thrombosis $(12.5 \%$ vs. $1.0 \%, p=0.002)$ compared with nonLMCA patients, while no difference was found between LMCA and non-LMCA in unplanned revascularization, stroke, bleeding, and MACCE (All $p>0.05$ ). Compared with non-LMCA patients, LMCA patients presenting with UA/ non-ST elevation myocardial infarction (NSTEMI) had higher incidence of MI (5.3\% vs. $1.8 \%, p=0.010)$, in-stent thrombosis $(3.1 \%$ vs. $0.8 \%, p=0.022)$, and stroke $(4.6 \%$ vs. $1.5 \%, p=0.016)$, while no difference was observed in allcause death, cardiac death, unplanned revascularization, bleeding, and MACCE (All $p>0.05$ ) (Table 4).

\section{Discussion}

In our study, patients presenting with ACS undergoing LMCA PCI was compared with ACS patients undergoing non-LMCA PCI in a large cohort of Chinese patients undergoing contemporary PCI. The main finding of this study is as follows: (1) in patients presenting with ACS, LMCAtargeted PCI is associated with higher risk of 2-year cardiac death, MI, in-stent thrombosis, and stroke. (2) Compared with non-LMCA-targeted PCI, LMCA-targeted PCI is an independent risk factor for 2-year MI. 


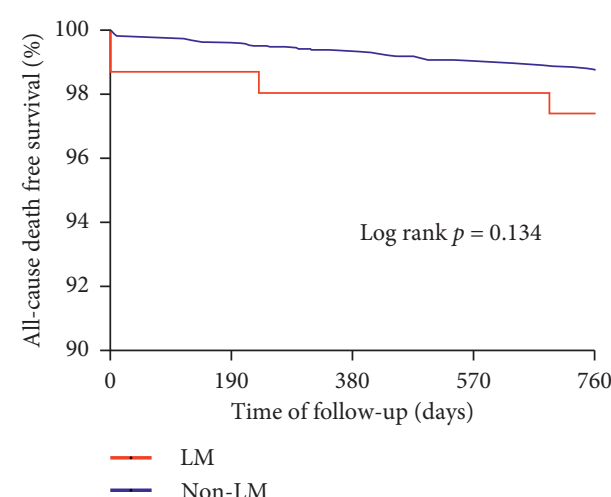

(a)

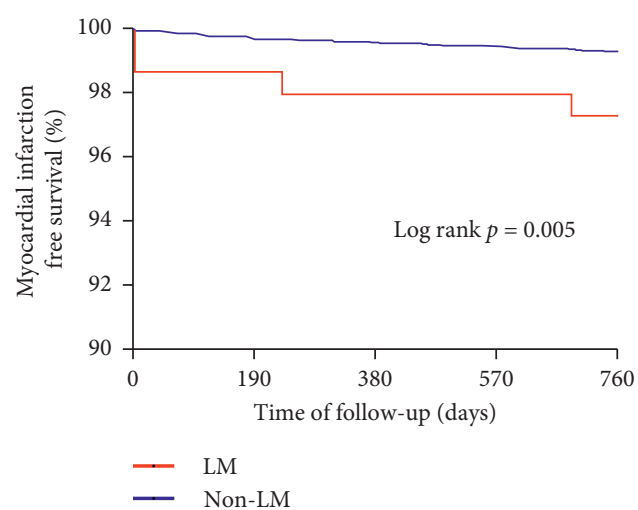

(c)

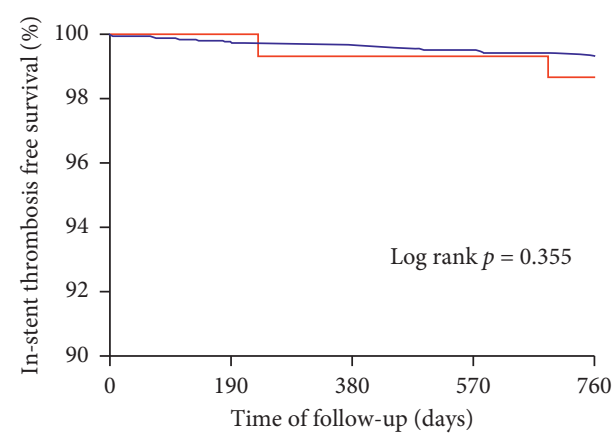

$\begin{array}{ll}- & \text { LM } \\ & \text { Non-LM }\end{array}$

(e)

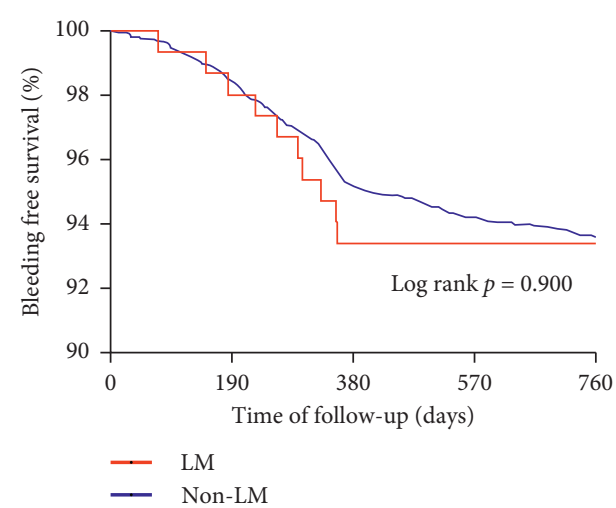

(g)

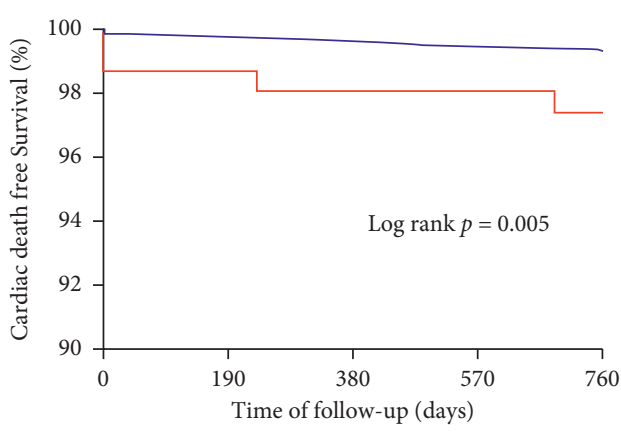

- LM

- Non-LM

(b)

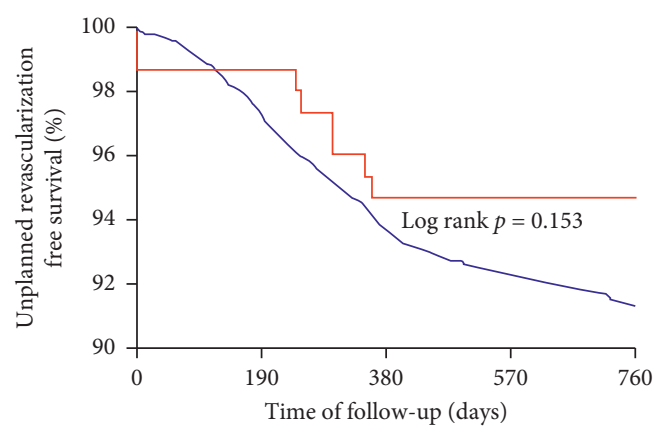

$\begin{array}{ll}- & \text { LM } \\ - & \text { Non-LM }\end{array}$

(d)

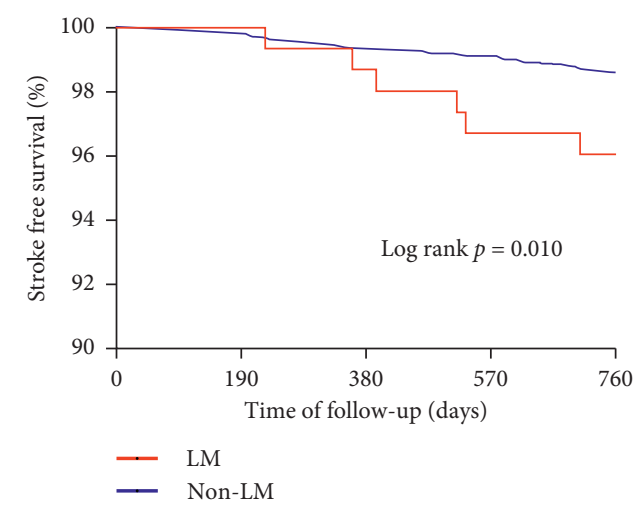

(f)

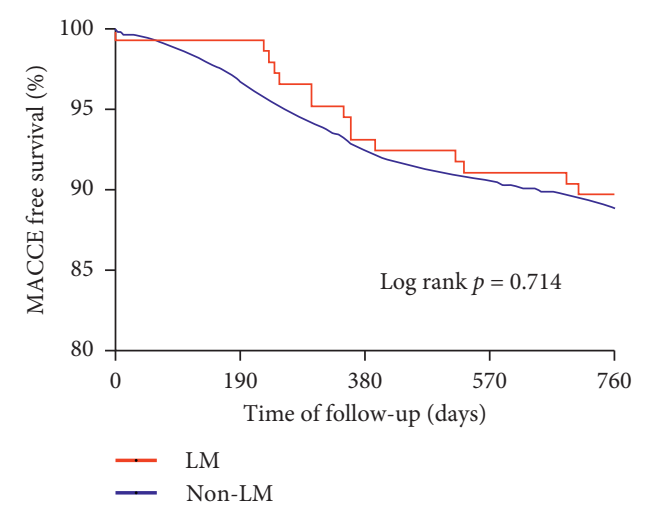

(h)

FIGURE 1: Kaplan-Meier survival curves on 2-year clinical endpoints between LMCA and non-LMCA groups for patients presenting with ACS. (a) All-cause death; (b) cardiac death; (c) myocardial infarction; (d) unplanned revascularization; (e) in-stent thrombosis; (f) stroke; (g) bleeding; (h) MACCE. MACCE = major adverse cardiac and cerebrovascular events. 


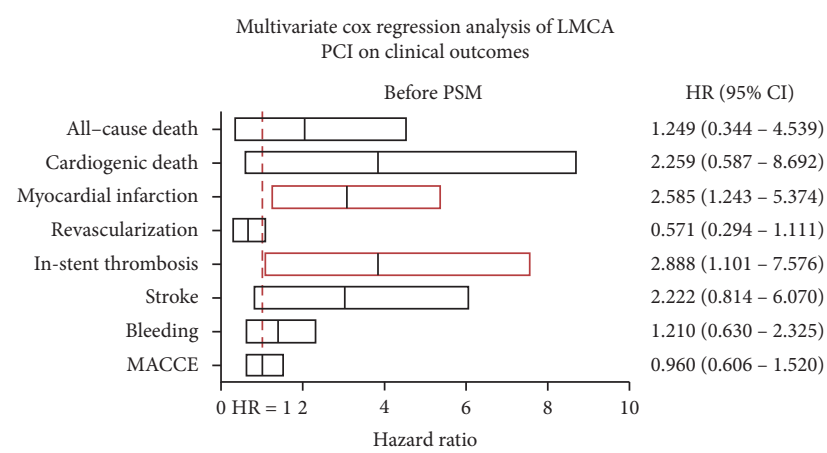

(a)

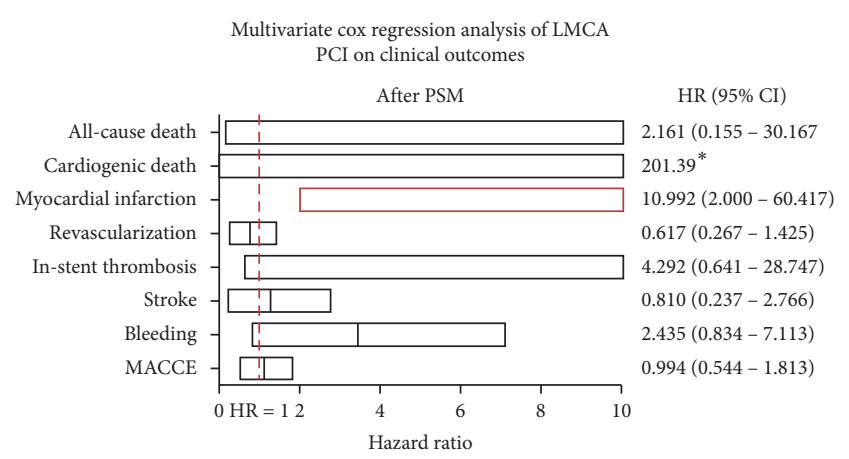

(b)

Figure 2: Adjusted hazard ratios of LMCA PCI for clinical outcomes. HR and 95\% CI of each clinical outcome are shown in the plot. Clinical outcomes shown in red bars are significantly different. (a) Before propensity score match; (b) after propensity score match. MACCE = major adverse cardiac and cerebrovascular event. Adjusted variables: LMCA, age, diabetes, hemoglobin, GFR, STEMI, UA, preprocedural SYNTAX score, puncture site, staged PCI, IVUS, IABP, successful PCI, and stent type. ${ }^{*}$ Data unavailable as no patient had cardiac death in the non-LMCA group after PSM.

TABLE 4: 2-year clinical outcomes of STEMI and UA/NSTEMI subgroups.

\begin{tabular}{|c|c|c|c|c|c|c|}
\hline & \multicolumn{3}{|c|}{ STEMI } & \multicolumn{3}{|c|}{ UA/NSTEMI } \\
\hline & LMCA $(n=24)$ & Non-LMCA $(n=1421)$ & $p$ value & LMCA $(n=131)$ & Non-LMCA $(n=4853)$ & $p$ value \\
\hline All-cause death & $3(12.5)$ & $28(3.0)$ & 0.013 & $1(0.8)$ & $54(1.1)$ & 1.000 \\
\hline Cardiac death & $3(12.5)$ & $19(1.3)$ & 0.005 & $1(0.8)$ & $28(0.6)$ & 0.539 \\
\hline Myocardial infarction & $4(16.7)$ & $31(2.2)$ & 0.002 & $7(5.3)$ & $85(1.8)$ & 0.010 \\
\hline Unplanned revascularization & $1(4.2)$ & $129(9.1)$ & 0.717 & $9(6.9)$ & $417(8.6)$ & 0.487 \\
\hline In-stent thrombosis & $3(12.5)$ & $14(1.0)$ & 0.002 & $4(3.1)$ & $37(0.8)$ & 0.022 \\
\hline Stroke & $0(0)$ & $16(1.1)$ & 1.000 & $6(4.6)$ & $72(1.5)$ & 0.016 \\
\hline Bleeding & $3(12.5)$ & $77(5.4)$ & 0.143 & $8(6.1)$ & $324(6.7)$ & 0.796 \\
\hline MACCE & $6(25.0)$ & $185(13.0)$ & 0.118 & $18(13.7)$ & $583(12.0)$ & 0.549 \\
\hline
\end{tabular}

STEMI = ST elevation myocardial infarction; NSTEMI = non-ST elevation myocardial infarction; $\mathrm{UA}=$ unstable angina; MACCE $=$ major adverse cardiac and cerebrovascular events.

For SCAD patients with LMCA disease undergoing revascularization, PCI is recommended in the $2018 \mathrm{ESC} /$ EACTS Guidelines on Myocardial Revascularization (Class I for low SYNTAX score and class IIa for intermediate SYNTAX score) [1]. In recent years, a number of randomized controlled studies have compared the long-term clinical outcomes of patients LMCA disease undergoing different revascularization procedures. The EXCEL [10] and PRECOMBAT [20] study showed similar rate of long-term adverse events between patients undergoing LMCA PCI or CABG, while NOBLE trial [21] findings suggested that CABG might still be a better option for these patients. However, little is known for the long-term effect of PCI in ACS patients with significant LMCA disease. Sim et al. found acute MI patients with a culprit LMCA having higher inhospital mortality than patients with nonculprit LMCA, while 1-year clinical outcomes were similar [22]. In a larger observational study [23], long-term survival rates (median follow-up time was 6.3 years) were similar between STEMI patients and UA/NSTEMI patients due to unprotected LMCA disease. The DELTA all-comer, a multinational registry-revealed PCI for ACS in ULMCA is associated with similar rate death, cerebrovascular accident, and MI compared with CABG at long-term follow-up [24]. Patel et al. [25] found unprotected LMCA occlusion in patients undergoing primary PCI is independent predictor of 30-day and 3-year all-cause mortality. To the best of our knowledge, there is no large-sample observational study available yet to compare long-term clinical outcomes of ACS patients undergoing PCI between LMCA and non-LMCA as the target vessel.

ACS patients with significant unprotected LMCA disease represent one of the most high-risk types of coronary artery disease, especially for patients with acute MI caused by LMCA culprit lesion. As LMCA supplies blood perfusion for the majority of left ventricle myocardium regardless of coronary artery dominance, acute LMCA infarction leads to large infarction area [26]. A large proportion of AMI patients with LMCA involvement present cardiogenic shock, who are at much higher risk of in-hospital and short-term mortality [27]. Unfortunately, cardiogenic shock data are unavailable in our cohort database, so this serious complication was not further analyzed in this study. It is worth noticing that in the LMCA group, the vast majority of patients had unprotected LMCA (94.8\%). With a patent bypass graft to distal coronary arteries, it is reasonable to infer ACS patients with protected LMCA have better prognosis compared with patients with unprotected LMCA. Thus, high proportion of unprotected LMCA in the LMCA group also contributed to worse clinical outcomes. 
Furthermore, our patients with significant LMCA disease are complicated by other characteristics and comorbidities leading to higher mortality, including higher age, lower GFR level, and more patients with diabetes. Finally, patients in the LMCA group had generally more complex coronary lesions compared with non-LMCA patients, as characterized by higher proportion of trivessel disease and higher level of preprocedural SYNTAX score. These factors all contribute to higher cardiac mortality in LMCA group in our study population, despite efforts to improve patient survival by less use of first-generation DES, more use of IABP support, IVUS guidance, and staged PCI to treat multivessel disease in the LMCA group. After adjustment of baseline characteristics that are significantly different across the groups using Cox regression analysis, however, the incidence of cardiac death and stroke between the groups was no longer significantly different, indicating the higher risk of 2-year cardiac mortality and stroke in the LMCA group can be attributed to patients' poorer general baseline condition.

Clinical presentation in our patient population is an important indicator for acute and long-term prognosis. Compared with less severe conditions like UA or NSTEMI, patients presenting with STEMI with LMCA culprit lesion are critically ill, often leading to abrupt circulatory failure, fatal ventricular arrhythmia, and sudden cardiac death [28]. Since STEMI with culprit LMCA often leads to sudden death before the patient reaches hospital, we see a significantly lower proportion of hospitalized STEMI patients in the LMCA group ( $15.5 \%$ vs. $22.6 \%, p=0.035)$, which is in accordance with a previous report [13]. Due to the heterogeneity of different clinical presentation, we further did a subgroup analysis on clinical outcomes of STEMI and NSTEMI/UA patients. Not surprisingly, results showed STEMI subgroup contributed more to higher 2-year allcause and cardiac mortality, MI, and in-stent thrombosis in the LMCA group.

There are several inherent limitations in our study. First, whether the lesion in LMCA is the culprit lesion causing ACS is unknown. We acknowledge that whether LMCA is the culprit vessel is an important factor determining the outcome of patients with ACS; thus, the findings of our study cannot be specifically extended to ACS patients with culprit LMCA lesions. Second, due to limited sample size in the LMCA group, statistical analysis is less reliable than largesample comparison. Despite using Cox regression analysis and PSM to adjust for unmatched baseline characteristics, potential unknown risk factors still exist. Third, IVUS results, postdilatation results, whether patients received emergency or selective PCI, position of lesion in LMCA, stenting technique for bifurcation lesions, and complications including cardiogenic shock are unknown. Finally, trials with longer follow-ups are needed to further confirm our findings.

\section{Conclusion}

In patients presenting with ACS, LMCA-targeted PCI is associated with higher risk of 2-year cardiac death, MI, instent thrombosis, and stroke. LMCA-targeted PCI is an independent risk factor for 2-year MI. Our findings could provide useful prognosis information to clinicians and ACS patients with significant LMCA lesion.

\section{Data Availability}

The clinical data used to support the findings of this study are available from the corresponding author upon request.

\section{Conflicts of Interest}

The authors declare that they have no conflicts of interest.

\section{Acknowledgments}

We would like to give special thanks to the staff in the Department of Cardiology and Catheterization Laboratory, Fu Wai Hospital, for their research contributions. This study was supported by grants from the Ministry of Science and Technology of the People's Republic of China (2016YFC1301301) and National Natural Science Foundation of China (81770365).

\section{References}

[1] M. Sousa-Uva, F. J. Neumann, A. Ahlsson et al., "2018 ESC/ EACTS Guidelines on myocardial revascularization," European Heart Journal, vol. 40, no. 37, p. 3096, 2019.

[2] T. Palmerini, P. Serruys, A. P. Kappetein et al., "Clinical outcomes with percutaneous coronary revascularization vs coronary artery bypass grafting surgery in patients with unprotected left main coronary artery disease: a meta-analysis of 6 randomized trials and 4,686 patients," American Heart Journal, vol. 190, pp. 54-63, 2017.

[3] N. Nerlekar, F. J. Ha, K. P. Verma et al., "Percutaneous coronary intervention using drug-eluting stents versus coronary artery bypass grafting for unprotected left main coronary artery stenosis: a meta-analysis of randomized trials," Circulation: Cardiovascular Interventions, vol. 9, no. 12, 2016.

[4] A. Chieffo, A. Tanaka, G. Giustino et al., "The DELTA 2 registry: a multicenter registry evaluating percutaneous coronary intervention with new-generation drug-eluting stents in patients with obstructive left main coronary artery disease," JACC Cardiovasc Interv, vol. 10, pp. 2401-2410, 2017.

[5] B. Vaquerizo, T. Lefevre, O. Darremont et al., "Unprotected left main stenting in the real world," Circulation, vol. 119, no. 17, pp. 2349-2356, 2009.

[6] E. Meliga, H. M. Garcia-Garcia, M. Valgimigli et al., "Longest available clinical outcomes after drug-eluting stent implantation for unprotected left main coronary artery disease," Journal of the American College of Cardiology, vol. 51, no. 23, pp. 2212-2219, 2008.

[7] E. Boudriot, H. Thiele, T. Walther et al., "Randomized comparison of percutaneous coronary intervention with sirolimus-eluting stents versus coronary artery bypass grafting in unprotected left main stem stenosis," Journal of the American College of Cardiology, vol. 57, no. 5, pp. 538-545, 2011.

[8] A. Chieffo, V. Magni, A. Latib et al., "5-year outcomes following percutaneous coronary intervention with drug-eluting stent implantation versus coronary artery bypass graft for unprotected left main coronary artery lesions," JACC: Cardiovascular Interventions, vol. 3, no. 6, pp. 595-601, 2010. 
[9] D.-W. Park, Y.-H. Kim, H. G. Song et al., "Long-term outcome of stents versus bypass surgery in diabetic and nondiabetic patients with multivessel or left main coronary artery disease," Circulation: Cardiovascular Interventions, vol. 5, no. 4, pp. 467-475, 2012.

[10] G. W. Stone, J. F. Sabik, P. W. Serruys et al., "Everolimuseluting stents or bypass surgery for left main coronary artery disease," New England Journal of Medicine, vol. 375, no. 23, pp. 2223-2235, 2016.

[11] A. Pappalardo, M. A. Mamas, F. Imola et al., "Percutaneous coronary intervention of unprotected left main coronary artery disease as culprit lesion in patients with acute myocardial infarction," JACC: Cardiovascular Interventions, vol. 4, no. 6, pp. 618-626, 2011.

[12] G. B. Pedrazzini, D. Radovanovic, G. Vassalli et al., "Primary percutaneous coronary intervention for unprotected left main disease in patients with acute ST-segment elevation myocardial infarction," JACC: Cardiovascular Interventions, vol. 4, no. 6, pp. 627-633, 2011.

[13] S. Obeid, N. Yousif, A. Schelldorfer et al., "Short-term outcome after left main interventions in patients presenting with acute coronary syndrome," The Journal of Invasive Cardiology, vol. 30, no. 30, pp. 98-104, 2018.

[14] F. Gao, Y. J. Zhou, Z. J. Wang, Z. X. Yan, X. L. Liu, and $H$. Shen, "Transradial coronary intervention versus coronary artery bypass grafting for unprotected left main and/or multivessel disease in patients with acute coronary syndrome," Angiology, vol. 67, no. 1, pp. 83-88, 2016.

[15] J. Y. Baek, S. M. Seo, H.-J. Park et al., "Clinical outcomes and predictors of unprotected left main stem culprit lesions in patients with acute ST segment elevation myocardial infarction," Catheterization and Cardiovascular Interventions, vol. 83, no. 7, pp. E243-E250, 2014.

[16] S. Doucet, E. M. Jolicœur, P. W. Serruys et al., "Outcomes of left main revascularization in patients with acute coronary syndromes and stable ischemic heart disease: analysis from the EXCEL trial," American Heart Journal, vol. 214, pp. 9-17, 2019.

[17] K. Thygesen, J. S. Alpert, A. S. Jaffe et al., "Third universal definition of myocardial infarction," European Heart Journal, vol. 33, no. 33, pp. 2551-2567, 2012.

[18] D. E. Cutlip, S. Windecker, R. Mehran et al., "Clinical end points in coronary stent trials," Circulation, vol. 115, no. 17, pp. 2344-2351, 2007.

[19] R. Mehran, S. V. Rao, D. L. Bhatt et al., "Standardized bleeding definitions for cardiovascular clinical trials," Circulation, vol. 123, no. 23, pp. 2736-2747, 2011.

[20] J. M. Ahn, J. H. Roh, Y. H. Kim et al., "Randomized trial of stents versus bypass surgery for left main coronary artery disease: 5-year outcomes of the PRECOMBAT study," Journal of the American College of Cardiology, vol. 65, no. 65, pp. 2198-2206, 2015.

[21] T. Mäkikallio, N. R. Holm, M. Lindsay et al., "Percutaneous coronary angioplasty versus coronary artery bypass grafting in treatment of unprotected left main stenosis (NOBLE): a prospective, randomised, open-label, non-inferiority trial," The Lancet, vol. 388, no. 10061, pp. 2743-2752, 2016.

[22] D. S. Sim, Y. Ahn, M. H. Jeong et al., "Clinical outcome of unprotected left main coronary artery disease in patients with acute myocardial infarction," International Heart Journal, vol. 54, no. 4, pp. 185-191, 2013.

[23] S. M. Gharacholou, N. N. Ijioma, R. J. Lennon et al., "Characteristics and long term outcomes of patients with acute coronary syndromes due to culprit left main coronary artery disease treated with percutaneous coronary intervention," American Heart Journal, vol. 199, pp. 156-162, 2018.

[24] S. A. Pyxaras, L. Hunziker, A. Chieffo et al., "Long-term clinical outcomes after percutaneous coronary intervention versus coronary artery bypass grafting for acute coronary syndrome from the DELTA registry: a multicentre registry evaluating percutaneous coronary intervention versus coronary artery bypass grafting for left main treatment," EuroIntervention, vol. 12, no. 5, pp. e623-e631, 2016.

[25] N. Patel, G. L. De Maria, G. Kassimis et al., "Outcomes after emergency percutaneous coronary intervention in patients with unprotected left main stem occlusion," JACC: Cardiovascular Interventions, vol. 7, no. 9, pp. 969-980, 2014.

[26] M. Sadowski, W. Gutkowski, G. Raczyński, A. JanionSadowska, M. Gierlotka, and L. Poloński, "Acute myocardial infarction due to left main coronary artery disease in men and women: does ST-segment elevation matter?" Archives of Medical Science, vol. 6, pp. 1197-1204, 2015.

[27] M. M. Vis, M. A. Beijk, M. J. Grundeken et al., "A systematic review and meta-analysis on primary percutaneous coronary intervention of an unprotected left main coronary artery culprit lesion in the setting of acute myocardial infarction," JACC: Cardiovascular Interventions, vol. 6, no. 4, pp. 317-324, 2013.

[28] M. S. Lee, P. Bokhoor, S.-J. Park et al., "Unprotected left main coronary disease and ST-segment elevation myocardial infarction," JACC: Cardiovascular Interventions, vol. 3, no. 8, pp. 791-795, 2010. 